\title{
Conociendo la historia de Colombia: la mata que "mata" y la otra cara de la
} moneda

\section{Knowing Something about the History of Colombia: The Plant that Kills and the other side of the coin}

\section{Por: María José Ortiz Torres ${ }^{1}$}

Recibido: 20-11-2012

\section{Resumen}

Colombia es considerado uno de los países con mayor influencia del narcotráfico en el mundo, con cuatro de los carteles de narcotráfico más significativos en el marco de la micro y macroeconomía y del desarrollo del conflicto colombiano. Las culturas indígenas de América del Sur han implementado las hojas de coca como parte de su cultura. Sin embargo, la economía del país ha declinado en todas las áreas de producción, por lo cual el uso y expendio de sustancias psicoactivas durante las décadas de 1960 y 1970 incrementaron en un gran porcentaje la macroeconomía en Colombia.

No obstante, se pusieron en marcha leyes de prohibición en Estados Unidos y en Colombia disparan el enfoque negativo de las drogas en la sociedad y llevan a castigar a quienes las cultivaran, comercializaran o distribuyeran en el país. Con este panorama, se pretende realizar un análisis al problema social que aqueja la sociedad colombiana en el marco de la campaña "Colombia territorio libre de droga" con base en el concepto de ambiente como un constructo social mediado por elementos culturales y representaciones simbólicas que están ideológica y políticamente condicionadas y que ejercen un papel decisivo en los modos de interpretar la vida cotidiana, de construir valores, de desarrollar conocimiento y demás saberes que hacen lectura y comprensión crítica del entorno, como parte de un ejercicio de reflexión del Seminario de Educación Ambiental en el Departamento de Biología de la Universidad Pedagógica Nacional

Palabras clave: narcotráfico, guerra, ambiente, cultura, conflicto social, interculturalidad

\section{Abstract}

Colombia is considered one of the most influential countries in the world of drug trafficking with four of the most significant drug cartels under the micro and macroeconomic and development of the conflict. Indigenous cultures of South America implemented the coca as part of their culture. However, the economy has declined in all areas of production therefore, the use and sale of psychoactive substances during 1960 and 1970 increased by a large percentage of macroeconomics in Colombia.

However it launched prohibition laws in the United States and Colombia dissipate the negative approach of drugs in society and punish those

\footnotetext{
${ }^{1}$ Representante de los estudiantes ante el Consejo Académico- Universidad Pedagógica Nacional periodo (2009-2011). Estudiante de Licenciatura en Biología. IX semestre. 2011. E-mail:

majoT 34@hotmail.com
} 
who cultivated, marketed or distributed in the country. With this background, it is intended to analyze the social problems afflicting the Colombian society in the framework of the campaign "Colombia drug-free zone" based on the concept of environment as a social construct mediated by cultural elements and symbolic representations that are ideological and politically conditioned and exercised a decisive role in interpreting the ways of everyday life, to build values, to develop knowledge and other knowledge that make reading and critical understanding of the environment as part of an exercise in reflection of the Seminar on Environmental Education Department of Biology at the Universidad Pedagógica Nacional

Key words: drug, war, environment, culture, social conflict, intercultural

\section{Introducción}

Desde el establecimiento de la guerra contra las drogas, los Estados Unidos y algunos países europeos han proveído al gobierno colombiano ayuda logística y financiera para implementar planes que permitan combatir el tráfico de drogas. El programa más notable ha sido el Plan Colombia, el cual también está destinado a combatir los grupos armados ilegales calificados por estos países como terroristas, entre ellos paramilitares y guerrillas, que en la década de 1980 empezaron a financiarse con esta actividad ilícita. Sin embargo, durante la década de los años 90, ya se venía pensando en la erradicación fuerte de cultivos en varias zonas de América del Sur como Perú y Bolivia. Se contempló inicialmente el consumo total de cocaína, estimado en 650 toneladas de las cuales 300 se consumen en Estados Unidos, 100 en Europa, 150 en América del Sur, incluidas 50 en forma de hoja de coca para consumo tradicional y las restantes en otros mercados (Programa de las Naciones Unidas para la Fiscalización Internacional de Drogas, Tendencias Mundiales de las Drogas llícitas, 2001.)

\begin{tabular}{|c|c|c|c|}
\hline \multicolumn{4}{|c|}{$\begin{array}{l}\text { RESULTADOS OPERACIONALES 2011*(1) } \\
\qquad(21 / 08 / 2011)\end{array}$} \\
\hline \multicolumn{4}{|c|}{ ERRADICACIÓN CULTIVOS ILÍCITOS (ha) } \\
\hline CULTIVO & $\begin{array}{l}\text { ASPERSIÓN } \\
\text { AÉREA }\end{array}$ & MANUAL & TOTAL \\
\hline COCA & $75.999,00$ & $16.399,30$ & $92.398,30$ \\
\hline AMAPOLA & & 283,24 & 283,24 \\
\hline MARIHUANA & & 90,90 & 90,90 \\
\hline TOTAL & $75.999,00$ & $16.773,44$ & $92.772,44$ \\
\hline
\end{tabular}

Cuadro tomado de: Dirección Nacional de Estupefacientes (DNE) 2011, estimativo de erradicación de cultivos ilícitos. Historia de los cultivos ilícitos en Colombia

Un fenómeno que preocupa enormemente a los gobiernos nacional y regional es la dinámica migratoria ocasionada por los cultivos ilícitos. En Colombia, las corrientes migratorias cambiaron el mapa demográfico de los territorios con mayor presencia de cultivos ilícitos, es así como la población de la Amazonía colombiana aumentó de 50.700 en 1983 a 502.876 (DANE, 2004) lo cual indica que en efecto se consideraría el negocio más rentable en países del trópico con producción de droga. 


\begin{tabular}{|l|c|c|c|c|c|c|c|c|}
\hline \multicolumn{7}{|c|}{ CULTIVOS ILÍCITOS EN COLOMBIA 2002-2009 (ha) } \\
\hline Coca & $\mathbf{2 0 0 2}$ & $\mathbf{2 0 0 3}$ & $\mathbf{2 0 0 4}$ & $\mathbf{2 0 0 5}$ & $\mathbf{2 0 0 6}$ & $\mathbf{2 0 0 7}$ & $\mathbf{2 0 0 8}$ & $\mathbf{2 0 0 9}$ \\
\hline Amapola & 102.071 & 86.340 & 80.350 & 85.750 & 77.870 & 98.899 & 80.953 & 68.025 \\
\hline Marihuana & 4.153 & 4.026 & 3.950 & 1.950 & 1.023 & 715 & 394 & 356 \\
\hline
\end{tabular}

Cuadro tomado de: Dirección Nacional de Estupefacientes (DNE) Año 2002-2009, estimativo de identificación de cultivos de coca en países productores. Historia de los cultivos ilícitos en Colombia

Cuando el estudio nacional sobre consumo de drogas reveló que Colombia ya presentaba indicadores preocupantes en materia de adicciones, el Gobierno y la sociedad civil se comprometieron de manera participativa a redoblar los esfuerzos para frenar y revertir la situación. A tono con las buenas prácticas en otros países, la política colombiana explícitamente se propone ampliar la oferta de servicios de prevención, tratamiento y rehabilitación. Los países que a duras penas han logrado estabilizar o reducir el consumo de drogas recientemente, le han apostado precisamente a esta estrategia, que además de reducir terribles dramas individuales, familiares y comunitarios, generan seguridad ciudadana, un bien público esencial para la paz y el desarrollo.

Aldo Lale-Demoz, representante de la Oficina de las Naciones Unidas contra la Droga y el Delito en Colombia, UNODC, abrió con su discurso el lanzamiento de la campaña "Colombia, territorio libre de drogas" el 8 de junio de 2010, liderada por el Ministerio del Interior y de Justicia y la Dirección Nacional de Estupefacientes, con el apoyo del Consejo Nacional de Estupefacientes. La campaña busca afianzar el compromiso personal, familiar y social, frente a la toma de decisiones saludables y la vida libre de elementos que la esclavicen, como las drogas.

En el discurso de esta campaña se hace alusión a que "las consignas genéricas, puramente enfocadas en el 'no a las drogas', han dado paso a mensajes propositivos, que invitan a construir una muralla social contra los efectos nocivos de éstas".

De ahí el mensaje "Comunidad libre", que hace parte de la campaña y cuyos primeros comerciales institucionales de televisión y radio, además de su página web, fueron presentados con un discurso de expresión de libertad de los jóvenes frente a las drogas en los diferentes territorios reales y simbólicos en los cuales se desenvuelve el individuo, como son la familia, los amigos, el barrio, los centros educativos, entre otros.

\section{Características de la campaña}

Con el objetivo de disminuir el consumo de droga en el país, de manera educativa, la División Nacional de Estupefacientes (DNE) prioriza la lucha contra las drogas como 
elemento estructural de las problemáticas de un conflicto social, económico y político. En la campaña se pueden hacer evidentes algunas premisas como:

1. De manera creativa y llamativa, se logra captar la atención en que el problema central es el cultivo realizado por numerosos sectores sociales que fomenta de manera sustancial el narcotráfico en Colombia.

2. La campaña fortalece de manera sustancial el programa de guardabosques, dirigiendo la atención del ciudadano hacia el deber del monitoreo y control constante de cultivos ilícitos.

3. Muchos de los videos lanzados a la red y medios de comunicación, donde se repite constantemente, "Comercia sonrisas, abrazos y besos", "Cultiva alegría, perdón y dignidad", son frases que ven de una forma superficial el conflicto no armado, sino social, político y económico que vive el país a diario, agregándole la categoría de capital humano a una nación que ha sufrido los golpes de una guerra profunda.

4. El campo se mira desde la óptica de producción feudalista y completamente capitalista y no se profundiza sobre el problema de reforma agraria, la distribución real de tierras que debe hacer la nación como primer paso para construir una nación a partir del respeto, la igualdad y la justica social

\section{Una mirada desde la historia colombiana}

Le estamos agradecidos al Washington post, New York Times, Time Magazine y a otras grandes publicaciones cuyos directores han asistido a nuestras reuniones y han respetado la promesa de discreción casi 40 años, ... nos habría sido imposible desarrollar nuestro plan para el mundo durante todos esos años si hubiéramos estado expuestos a la luz pública. Pero el mundo es mucho más sofisticado y está preparado para marchar hacia un gobierno mundial. La soberanía supranacional de una elite intelectual y de los banqueros mundiales es ciertamente preferible a la autodeterminación nacional practicada en los últimos siglos. (VEGA, 1997. p. 149)

David Rockefeller, citado por Bruce Katz y René Silva, "La mundialización y la candidez del académico", en Eco portal, noviembre 15 de 2004

\section{¿Narcotráfico en Colombia?}

El problema del narcotráfico en Colombia no es un problema de unas plantas que generan una entrada fácil de dinero a grupos al margen de ley. El problema del narcotráfico viene de hace más de 50 años y su punto álgido se expresa en un conflicto social, político y económico nacional e internacional, al que regímenes estatales y paraestatales han dado cabida en Colombia hasta la actualidad. De forma paralela, mientras en Colombia se fortalecían en un segundo plano los ingresos a través de las transnacionales, en las potencias se gestaba el fortalecimiento del capitalismo bárbaro a través de los discursos ambientalistas, en donde claramente defienden acciones conservacionistas ficticias disfrazando la realidad del ambiente 
versus la acumulación de capital, a través de lo que se conoce como el mal llamado ecoturismo, claro ejemplo de ello es la línea de hoteles Decamerón.

Remontándonos en la historia, durante la Segunda Guerra Mundial se demostró lo indescriptible: la glorificación de la revolución industrial. Con ello, las empresas transnacionales establecen su papel protagónico acerca de lo político, económico y social en la historia del mundo con negocios de segunda mano (Observatorio Social de Megaproyectos, Transnacionales y Derechos Humanos, 2008). Con la caída del muro del Berlín, en 1989, se expande la ola transnacionalista de las grandes potencias mundiales. La Unión Europea y EE.UU. logran extenderse en los territorios ocupados por los países que conformaban la Unión de Republicas Socialistas Soviéticas -URSS, incluyendo la guerra de Vietnam con la llamada guerra de guerrillas, conocidas como "Vietcong", dando paso a la devastadora globalización en los países "tercermundistas". Con el discurso conservacionista del ambiente, más de una transnacional logró acceder, sobre los años 80 y 90, a los recursos naturales de muchos países suramericanos como Colombia. Durante su gobierno, Richard Nixon fue reelegido bajo la promesa de acabar con la drogadicción, lo cual nunca ocurrió. En vista de ello, el estado formó la Drug Enforcement Agency, DEA, con 200 millones de dólares para "acciones encubiertas". Un analista americano, Barrington Moore, explicó la situación en 1978 al señalar el espaldarazo que la represión brindaba al incremento de los precios y a la utilidad de los traficantes. La corrupción era sutilmente pagada por las mafias del tráfico, del lavado de dólares, de la venta de químicos y armas, que tenían ciudadanía norteamericana (Moore, 1989).

De esta manera, la economía ilegal basada en la marihuana, la cocaína y la amapola creció en distintas regiones marginadas. Empresarios extranjeros establecieron una alianza con el paramilitarismo para defender sus negocios, acaparar tierras y afianzar sus poderes locales. La implantación de políticas neoliberales le abrió camino a la explosión de este nuevo capitalismo de naturaleza ilegal que llevó a que estos productos se convirtieran en el principal motor a través del cual Colombia se insertaría en la globalización económica.

Así, llega el negocio del narcotráfico a Colombia. Como procesos paralelos pero bastante cercanos, la transformación de la planta de coca al alcaloide clorhidrato de cocaína, o del Cannabis sativa a la comúnmente conocida marihuana, resulta ser un negocio implantado por las grandes potencias para obtener mayores ingresos en sus flujos económicos. En 1978, Estados Unidos conoció el determinante influjo de la cocaína en la sociedad de la Florida. Los jefes de la droga empezaron a disputar el comercio de su producto y la distribución al por mayor en las grandes capitales. Miami fue testigo de una guerra que involucró a su policía corrupta y que dejó más de un centenar de asesinatos. Los intermediarios y traficantes del exilio cubano llevaron la peor parte y los colombianos se quedaron sin discusión con el reinado; integraron sus operaciones y captaron utilidades que, reinvertidas, permitieron expandir y dinamizar su producción y exportación. En 1976 la DEA calculaba entre 14 y 19 las toneladas métricas de cocaína que entraron de contrabando a Estados Unidos (Tokatlián y Bagley, 1990).

En los 80, figuras como los hermanos Rodríguez Orejuela, los hermanos Gacha y Pablo Escobar, dieron inicio a este negocio con fines lucrativos personales que generaban redes con ciudadanos estadounidenses que pertenecían a grandes trasnacionales gringas, con el discurso de "les vamos a enseñar a explotar los recursos a los países subdesarrollados". Así, se generaron los famosos carteles del narcotráfico, que comenzaron a través de estas redes a despojar a campesinos de sus tierras, pagando el valor de la mano de obra a bajos precios y con los capos y los 
grandes funcionarios de empresas trasnacionales llevándose y controlando la economía nacional y por ende el manejo del país al servicio de las potencias.

En el país, se gestaban fuertes alianzas entre parlamentarios colombianos que comenzaron a ver con ojos ambiciosos el negocio del narcotráfico y dejaron permear el congreso con ello. El famoso Bogotazo fue una muestra clara y contundente que paralizó al país en 1948. El asesinato de Luis Carlos Galán por denunciar nexos del senador Alberto Santofimio con el narcotraficante Pablo Escobar al recibir lo que en aquel entonces se conocía como "dineros calientes", fue todo un acontecimiento público. Esto, generó el principio del gran exterminio de los carteles de Colombia, como el cartel de Cali o el de Medellín, y dio paso para que el negocio del narcotráfico no solo interesara a algunos sino a muchos que buscaban viabilidad económica.

Durante los años 1995 hasta 1997 se da la implantación local de las Convivir, cooperativas de seguridad privada, servicios comunitarios de vigilancia creados por el Gobierno Nacional mediante el Decreto 356 de 1994 y una resolución del 27 de abril de 1995 de la Superintendencia de Vigilancia y Seguridad Privada como medidas de seguridad para reforzar la seguridad frente a los actores del conflicto armado colombiano. Human Rights Watch (1998) sostuvo que un elemento crítico de la confusión en la distinción entre civiles y combatientes es el alistamiento de civiles anónimos que operan sin uniformes e insignias visibles en vehículos sin identificación. De hecho, el gobierno se aprovecha de este anonimato al permitir que las Convivir realicen sus operaciones en áreas residenciales, como en el caso de la Convivir de Rionegro. Hacia el final de su mandato en la Gobernación de Antioquia, Álvaro Uribe Vélez tiene bajo su responsabilidad la masacre de El Aro, por la cual fue cuestionada su administración, dando paso a la gesta del proceso de paramilitarismo en Colombia, a partir de las Convivir, con la creación de las AUC en 1997.

El vínculo entre las potencias mundiales bajo las operaciones de agentes de la DEA y de vínculos paramilitares promovido desde la década del sesenta por el gobierno de EEUU, legal y fácticamente implementado por el Estado colombiano, ha servido para desviar su responsabilidad en la secuencia sistemática de crímenes de lesa humanidad y para asegurar la impunidad (Decreto 3398 de 1965, Ley 48 de 1968 y Decreto 356 de 1994). El narcotráfico se consolida en la nación con mayor fuerza como un negocio lucrativo, que es ilegal para muchos, legal para unos cuantos y clandestinos para los que su vida dependía de ello. Los paramilitares, bajo la consigna de proteger a la nación de grupos guerrilleros, necesitaban fortalecerse y abastecerse económicamente. A partir de un discurso moralista, ambientalista y democrático, se justifican los altos índices de desplazamiento, desaparición forzada, muertes, extorsiones, inequidad en las tierras y altos índices de pobreza en la población colombiana, que ha durado por mas de 50 años.

En esta lógica paramilitar entran las trasnacionales con un desaforado afán de convertir a los países "tercermundistas" en locomotoras que trabajen sin parar, en la acumulación de riqueza extranjera trabajando bajo los recursos propios de donde no solo se desprende este amplio y basto tema del narcotráfico sino de los múltiples cultivos ilícitos que agudizan problemas de fondo en la historia colombiana. "No puede decirse, por completo, que la responsabilidad de todo ello recaiga sobre el narcotráfico. La realidad de Colombia es bastante compleja y son muchos los actores y escenarios que se cruzan en su construcción. Los estudios, además, son todavía frágiles. El problema del narcotráfico no ha sido tratado en forma clara y suficiente. El peligro que se cierne sobre todos aquellos que intenten acercarse al tema es real. (Atehortúa Cruz y Rojas Rivera, 2008). 
¿Cuál es el problema estructural de Colombia? ¿Las concepciones de lo que es realmente la droga?

El problema estructural en Colombia no radica en la existencia de cultivos de coca, amapola y marihuana. En el marco de la transformación de un ambiente basado en un constructo social mediado por elementos culturales y representaciones simbólicas que están ideológica y políticamente condicionadas y que ejercen un papel decisivo en los modos de interpretar la vida cotidiana, el pueblo se ha acostumbrado a obviar la memoria histórica de un país que se escuda en excusas superficiales sobre el problema estructural de un conflicto de hace muchos años. Los colombianos hemos construido un ambiente de conflicto como si fuese algo particular y muy propio, se torna un contexto tan normal que en la actualidad son pocos los que pueden realizar un análisis profundo de lo que enmarca realmente el conflicto colombiano. La actividad económica de los cultivos de coca en las zonas de colonización campesina de Colombia es el resultado de la convergencia en estos territorios de problemas políticos, económicos y sociales. Es ahí donde se teje una pirámide de flujos culturales en donde la coca para un indígena no representa lo mismo para un campesino, y por ende su relación económica gira de forma diferente. El desplazamiento, apoderamiento de territorio por la fuerza, las relaciones feudales por mantenerse en un área, reducción del terreno y maltrato ecológico, la pobreza, la concentración de tierras, violencia y crisis agraria fueron y siguen siendo factores determinantes para que muchos colombianos de las zonas andinas migren hacia la periferia, en busca de nuevas alternativas de vida (García Hoyos, 2002) entre otras, son consecuencias de una economía feudalista-narcotraficante que viven algunos sectores de Colombia.

Esta actividad permanece porque existen problemas estructurales irresueltos en el campo colombiano y fundamentalmente porque existe un capital internacional del narcotráfico que demanda la materia prima de la hoja de coca. En la génesis del problema están el conflicto agrario colombiano, la concentración de la tenencia de la tierra, la expulsión y desplazamiento de masas campesinas, la irrupción de la apertura neoliberal, su modelo de desarrollo excluyente, el aumento del consumo de drogas en el mundo y la coyuntura, desde los años 70 , del tráfico y el consumo de cocaína muy diferente a la coca desde tiempos precolombinos.

Estos elementos, que hacen parte de la dinámica del conflicto político, social y armado colombiano, igualmente explican la ocurrencia de los cultivos de coca como una actividad de resistencia y de economía campesina, así como el auge del narcotráfico y sus impactos en todas las esferas de la vida política y económica de la sociedad colombiana.

Con la apertura económica del presidente Cesar Gaviria a principios de los noventa, que buscaba incluir a Colombia en las políticas globalizantes del capitalismo y ampliar las posibilidades de mercado para la nación, se introduce el concepto de competencia extranjera. Los precios de muchos productos nacionales habían incrementado con el tiempo, y en un afán de poder entrar a las lógicas del mercado, muchos sectores productivos y empresariales desaparecieron. La reforma a los planes de vivienda, que se convirtió en un verdadero fracaso por los costos reales de esta en Colombia, que a raíz de las grandes inversiones hechas por el narcotráfico, encareció enormemente los precios en las construcciones nuevas y se reemplazó la vivienda de interés social por suntuosas construcciones a costos exorbitantes, otro factor de desplazamiento y búsqueda de nuevos ingresos para la población. La depresión del sector agrícola, en 1993, llevó la economía en este sector prácticamente a una recesión. Esto se debió al desmonte de los altos niveles de protección del sector, sumado a los bajos precios 
internacionales. Sumado a esto, el discurso de la "guerra contra las drogas" con el cual los Estados Unidos justifican la intervención política y militar en el conflicto colombiano, a través del Plan Colombia, esconde intereses que trascienden el proceso de producción, tráfico y consumo de estupefacientes, y que tienden a garantizar el flujo continuo de recursos estratégicos como el petróleo y al establecimiento de un orden continental neomercantilista mediante la implementación del Tratado Bilateral de Libre Comercio y del Área de Libre Comercio de las Américas.

Las fumigaciones indiscriminadas con glifosato han afectado gravemente la economía campesina, el estado de salud de los pobladores rurales y están produciendo impactos ambientales impredecibles al fumigarse selvas nativas, ríos, quebradas, ciénagas y humedales en ecosistemas estratégicos.

De lo verificado en la región del valle del río Cimitarra, en el Magdalena Medio, se ha evidenciado que por cada hectárea de coca fumigada se fumigaron en promedio cuatro hectáreas más de selvas, cuerpos de agua y cultivos de pan coger. Si bien es cierto que los cultivos extensivos de coca perjudican notablemente algunas áreas selváticas, y que en el proceso de producción de cocaína muchos químicos sobrantes son vertidos en los ríos, estos daños no se pueden comparar con los que ocasionan las denominadas "fumigaciones de erradicación" implementadas hace mucho tiempo por los gobiernos de la región y auspiciadas por los Estados Unidos (García Hoyos, 2002), destruyendo a su paso materiales orgánicos, químicos y microorganismos que ayudan en el proceso de nitrificación, e incluso aumentando problemas de carácter congénito en las poblaciones y sus habitantes.

Antes de la coca la deforestación en las selvas era más rápida, la siembra de cultivos, la venta de parcelas y el reinicio de la siembra. De esta manera, el colono deforestaba la selva, sembraba el maíz, la yuca y el plátano y luego de tres cosechas estaba obligado a "sembrar pasto", pues la tierra no daba más y los ingresos por la venta de los productos no permitían la inversión en agroquímicos, por lo que se veían obligados a vender las tierras colonizadas a los latifundistas o comerciantes que luego de endeudar a los campesinos, les compraban las fincas para extender sus haciendas (García Hoyos, 2002). De esta forma el campesino tenía cómo sostenerse y generar nuevos ingresos a partir de químicos, razón por la cual un sector considerable de población desplazada se ha incluido a generar mecanismos de producción de droga. El desconocimiento de las condiciones materiales en las que la población colombiana ha tenido que subsistir bajo unas situaciones de conflicto social, ha permeado la justificación de uso de métodos proambientalistas, desconociendo una realidad que se vive día a día en la relación que establece el hombre con su entorno natural.

Las estrategias del Plan Colombia al proponer establecer el control militar en el sur del país para erradicar los cultivos de droga, destruir las instalaciones de procesamiento, poner millones de dólares a la mano de Colombia y mejorar la interceptación de narcóticos en las vías terrestres, aéreas, marítimas y fluviales, constituyen una causa más del desplazamiento forzado, que se suma al accionar militar y paramilitar, al establecimiento de bloqueos alimentarios y sanitarios a zonas campesinas, haciendo mucho más precarias y pauperizando las condiciones de vida de las comunidades rurales. Las consecuencias de esta relación desigual, de un pueblo colonizado y un pueblo colonizador, donde quien tenga más acumulación de capital decide por encima de todos y de todo, van más allá del campo concreto del Plan Colombia: a lo largo del verano de 2002, Colombia se vio "obligada" a firmar acuerdos bilaterales con Estados Unidos en el marco del Tribunal Penal Internacional (cuyo estatuto ha sido ratificado 
por Colombia pero no por Estado Unidos), puesto que Washington había insistido en condicionar a esta firma su apoyo económico. (Capozzio, 2003)

Estas acciones ilegitiman todavía más al actual Estado colombiano, con una nueva figura de poder, Juan Manuel Santos, y lo alejan de sus ciudadanos; contrario a lo esperado, fortalecen a las guerrillas, y lo más grave, no solucionan el problema del narcotráfico. Curiosamente, al no solucionarse el problema ni nacional e internacionalmente, se está manteniendo un negocio que según datos preliminares, bordea cerca de los 500 mil millones de dólares anuales.

Cabe entonces preguntarse, si al campesino el narcotráfico le paga $\$ 2 ' 400.000$ (800 euros) por kilo de base de coca (no de cocaína), ¿quién entonces se está quedando con todo el valor agregado en la cadena del narcotráfico? ¿Y dónde se está invirtiendo este dinero? Solo en el 2001 Colombia exportó 839 toneladas métricas de cocaína, según datos del Transnational Institute (TNI). Ahora ¿realmente el problema está en el simple problema de que exista la planta como ser vivo o será la transformación y sus componentes químicos usados indiscriminadamente para someter a través de los medios de producción a un pueblo ignorante de su contexto?

Las razones fundamentales que Ilevaron a Colombia a ser epicentro del tráfico de droga, pueden resumirse en su extraordinaria posición geográfica con múltiples fronteras casi inexpugnables por la naturaleza, su acceso a los Océanos Pacífico y Atlántico, sus tradicionales rutas de contrabando tan antiguas como la Colonia, y un contrabando que tuvo incluso un gran apogeo con producto insignia del país: el café. (García Hoyos, 2002)

\section{En Colombia sí existen situaciones alternas y nada distantes}

¿Por qué no hablar de la comercialización de la palma de cera y de las mismas implicaciones que tiene el comercio de la coca con narcoparamilitares y paraestatales en Colombia? El negocio de la droga es mucho más lucrativo y más poderoso que el comercio del aceite de palma de cera. Sin embargo, se mueve bajo unas mismas condiciones políticas, sociales y económicas e incluso culturales que permean el país con una mentalidad de terrorismo y desolación.

La palma de cera africana es otro de los vivos ejemplos del discurso doble, hipócrita, interesado y moralista en el marco ambientalista, del cual se han aprovechado unos para poder generar mayores ingresos sin importar las consecuencias que llegan a agravar el conflicto armado en Colombia. El paramilitarismo, la corrupción, los terratenientes, el latifundismo en Colombia han hecho de la conservación un discurso interesado de ingresos seguros para unos cuantos, causando pobreza, hambre, desplazamientos forzados en muchas familias del Pacífico colombiano a cuenta de unos pesos y, en algunos casos, ningún ingreso por sus tierras y por cuidar los cultivos de palma y su transformación para la posterior comercialización y garantizar el lucro de los más adinerados del país

\section{Desde la educación ambiental}

La educación ambiental vista como un proceso formativo que posibilita la construcción de valores, actitudes, toma de decisiones favorables a la resolución de problemáticas ambientales frente al entorno que rodea a un grupo de personas, entendiendo que 
para obtener estos resultados debe haber un desarrollo colectivo e individual referente a problemáticas ambientales desde aspectos que son interdependientes como lo económico, político, social, biofísico y cultural, debe reconocer las relaciones que se tejen entre los sistemas sociales y naturales que se medían a través de la cultura.

El Estado colombiano no reconoce bajo este planteamiento las relaciones que puede establecer el ser humano consigo mismo, con otros y con el entorno que lo rodea. El discurso de conservación no puede ser manipulado ni mucho menos desangrado por los meros intereses económicos y políticos de la clase burguesa de este país. La campaña que lanza el gobierno nacional, desconoce por completo y en su estructura, un conflicto social, político y económico, así como los índices de pobreza, hambre, desplazamiento y estigmatiza la mata de coca, marihuana y amapola como las causantes del problema del narcotráfico en Colombia, cuando realmente este problema refiere a un conflicto que viene de años atrás y solo se solucionará con una política que permita la amplia y justa distribución de tierras en el país.

Empleando esta campaña que cosifica al campesino, al empresario, al estudiante, al maestro, a las amas de casa, padres de familia, poniendo de primer plano que el problema del consumo de drogas en adolecentes radica en el problema de la existencia de la planta de coca, marihuana o amapola. Realmente el consumo de drogas refiere a un aspecto pedagógico que es importante trabajar desde la consigna de consumo consciente, donde el joven colombiano conozca las concepciones y los debates que giran en torno al consumo desde sus concepciones, las implicaciones biológicas, sociales, económicas, políticas que acarrean en el marco de un conflicto social que vive nuestro país.

El gobierno nacional tiene dos opciones para poder erradicar el problema del narcotráfico en Colombia sin necesidad de desdibujar el problema del conflicto social que invade el país desde hace años, como lo hace con estas campañas e intentando evadir realmente la situación política y social del país. De un lado, la legalización de la hoja de coca y su uso tradicional, cultural y medicinal, la legalización de las drogas y el tratamiento de salud pública a los drogadictos; de otro lado, la solución está en el plano de las reformas estructurales que garanticen el acceso a la tierra mediante una reforma agraria integral que elimine el latifundio improductivo, que asegure el mercado interno para la producción agropecuaria nacional mediante un modelo de desarrollo incluyente y redistributivo.

Estas propuestas son parte de la solución al conflicto político, social y armado, de la justicia social en el campo y de la paz. Podrán contemplarse otras opciones que desde la concepción de vida y del contexto colombiano muchos maestros tengan la opción de poder tantear. Sin embargo, solo me queda preguntarle a cada maestro, cada estudiante, cada sujeto: ¿Es la planta, la droga o el narcotráfico el problema estructural de Colombia? Usted... ¿qué opina?

¿Cuando un pueblo enérgico y viril llora, la injusticia tiembla! - Fidel Castro 


\section{BIBLIOGRAFÍA}

- Dirección Nacional de Estupefacientes - DNE (2011). Estimativo de identificación de cultivos de coca en países productores. Historia de los cultivos ilícitos en Colombia

- Decreto 3398 de 1965, Ley 48 de 1968 y Decreto 356 de 1994.

- MOORE, Barrington. (1989). La injusticia: bases sociales de la obediencia y la rebelión. México, Universidad Nacional Autónoma de México.

- GARCÍA HOYOS, Juan Carlos. (2002). De la coca a la cocaína: una historia para comprender.

- HUMAN RIGHTS WATCH. (1998). Guerra sin cuartel. Colombia y el Derecho Internacional Humanitario.

- Observatorio Social de Megaproyectos, Transnacionales y Derechos Humanos. (2008) Las huellas del capital transnacional en Colombia. Bogotá, Colombia.

- Plataforma Colombiana de Derechos Humanos, Democracia y Desarrollo. (2008). Sin democracia, sin derechos: Quinto año de Gobierno de Álvaro Uribe Vélez. Ediciones Antropos Ltda., Bogotá, Colombia

- Programa de las Naciones Unidas para la Fiscalización Internacional de Drogas, Tendencias mundiales de las drogas ilícitas (2001) Bogotá, Colombia.

- TOKATLIÁN, Juan y BAGLEY, Bruce (1990). Economía y política del narcotráfico. Bogotá, Uniandes - Cerec.

- VEGA CANTOR, Renán. (2007) Un mundo incierto, un mundo para aprender y enseñar. Colección de ciencias sociales. Universidad Pedagógica Nacional. Editorial Nomos. Bogotá, Colombia.

- Versión online [http://historiayespacio.com/rev31/pdf/Rev 31 - / El narcotráfico en Colombia. Pioneros y capos/ Adolfo León Atehortúa Cruz, Diana Marcela Rojas Rivera]

- Versión online [http://www.cafebabel.es/article/10380/la-disputa-neocolonialsobre-colombia.html/ La disputa neocolonial sobre Colombia/ Chiara Capozzio] 